\title{
RESEARCH HIGHLIGHT Combating pancreatic cancer during its Rip Van Winkle sleep
}

\author{
Kıvanç Görgülü (D) ${ }^{1}$, Marina Lesina ${ }^{1}$ and Hana Algül ${ }^{1}$ \\ Cell Research (2020) 30:637-638; https://doi.org/10.1038/s41422-020-0348-z
}

\begin{abstract}
In a recent paper published in Cell, Ruscetti et al. showed that therapy-induced senescence in pancreatic ductal adenocarcinoma (PDAC) rewires vascular network within the cancer, which in turn makes tumor susceptible to chemotherapy. Moreover, senescence-associated secretory phenotypedependent activation of cancer vasculature increases $T$ cell invasion into PDAC structures, thereby allowing better outcome of immunotherapy.
\end{abstract}

Senescence is compromised in the system of physiologic and malignant events embracing cancer and cancer-related compartments. The heterogeneous cellular texture of cancer during its development and progression admonishes cellular senescence by driving senescence-associated secretory phenotype (SASP), intervening crosstalk with surrounding cells such as host normal cells, immune effector cells and endothelial cells. ${ }^{1}$ Detrition of telomeres, induction of Ras downstream effectors, or therapeutic approaches can trigger SASP in cancer cells, which in turn unleashes chain reactions in the tumor microenvironment (TME). ${ }^{1}$ Since anticancer regimens induce senescence in cancer, the pivotal role of senescence-related properties became an interesting topic in cancer field including pancreatic ductal adenocarcinoma (PDAC). ${ }^{2}$ The emerging knowledge of the occurrence of senescence in PDAC showed that this cellular state may enter in force beginning with preneoplastic lesions, mutational status of the tumor, inflammation-driven carcinogenesis and cancer initiation steps. ${ }^{3-7}$ Finally, the dismal prognosis of PDAC patients urges the scientific community to open new therapeutic windows and widen the horizons in the treatment of PDAC. Committed to these challenges, Ruscetti et al. reported in a recent Cell paper that therapy-induced senescence in PDAC rewires the vascular network within the cancer, creating susceptibility of the tumor to chemoand immunotherapeutic agents.

In this study, Scott Lowe's group took advantage of previous studies and generated a comprehensive picture of already known puzzle pieces in the field of cancer. Inducing senescence by targeting MEK (trametinib) and CDK4/6 (palbociclib) modifies the TME to allow new flanks to increase efficacy of chemotherapy and immunotherapy in PDAC $^{8}$ (Fig. 1). The combined treatment modality of trametinib and palbociclib (T/P) decreased proliferation of cancer cells via inhibition of ERK and RB phosphorylation, leading to senescence induction in endogenous murine tumor and orthotopic transplantation models. Interestingly, the combo treatment reorganized TME architecture towards increased vasculature and decreased hyaluronic acid without altering collagen deposition or accumulation of a-smooth muscle actin (aSMA)-positive fibroblasts. Unlike reported in previous studies, these changes were not associated with increased metastasis. ${ }^{9}$ On the contrary, metastatic dissemination of cancer cells was attenuated. These effects were mediated by SASP factors including pro-angiogenic factors and matrix metalloproteases released from cancer cells exposed to combo treatment. As previously shown, the secretion of SASP factors depends on the NF-KB/RelA pathway. Most importantly, comprehensive experiments outlined in the study successfully pinpointed VEGF/ICAM as the most relevant axis in vascular remodeling, endothelial activation and improved perfusion allowing the immune system to access pancreatic cancer core. This scenario improved the responsiveness of the tumor to the treatment with chemotherapy (gemcitabine) and immunotherapy (PD1).

Surprisingly, the tumor vasculature following combo treatment failed to improve hypoxic conditions within the tumor. Activation of $\mathrm{CD}^{+} \mathrm{T}$ cells was not per se sufficient to demonstrate effective anti-tumor response in the context of combo treatment. Exhausted immune response, however, was resuscitated by additional PD-1 checkpoint blockade to reinvigorate anti-tumor immunity. Eventually, PD-1 therapy increased SASP-associated tumor-immune equilibrium, incrementally decreasing tumor volume and improving survival.

Although this study highlights the options to increase chemotherapy sensitivity and to convert a cold tumor to a hot one, clinical activities to transfer this concept into the bedside raise important concerns. Firstly, safety and tolerability of continuous combination of trametinib and palbociclib along with immune-oncology will pose a significant challenge. In the context of still existing hypoxic conditions, tumor cells may likely overcome combo treatment-induced senescence through biallelic TRP53 or INK4A mutation/deletion and will gain more invasiveness facilitated by modified stromal architecture and the presence of increased repertoire of matrix metalloproteases. The negative results of the HALO-301 clinical trial testing PEGPH2O in metastatic PDAC patients increased the awareness towards targeting TME without exact understanding of complexity in intra-tumoral dynamics. ${ }^{10}$ Nonetheless, this very interesting study reveals mechanistically how to attack stroma and the tumor cells at the same time, opening a new flank for immunotherapy.

In conclusion, this study provides an important aspect for combinatory therapeutic approaches associated with senescence induction. One thing has to be kept in mind: This Rip van Winkle sleep is volatile. ${ }^{11}$ Through overcoming the state of senescence, PDAC cells may regain their aggressiveness and would go back even stronger to work. 


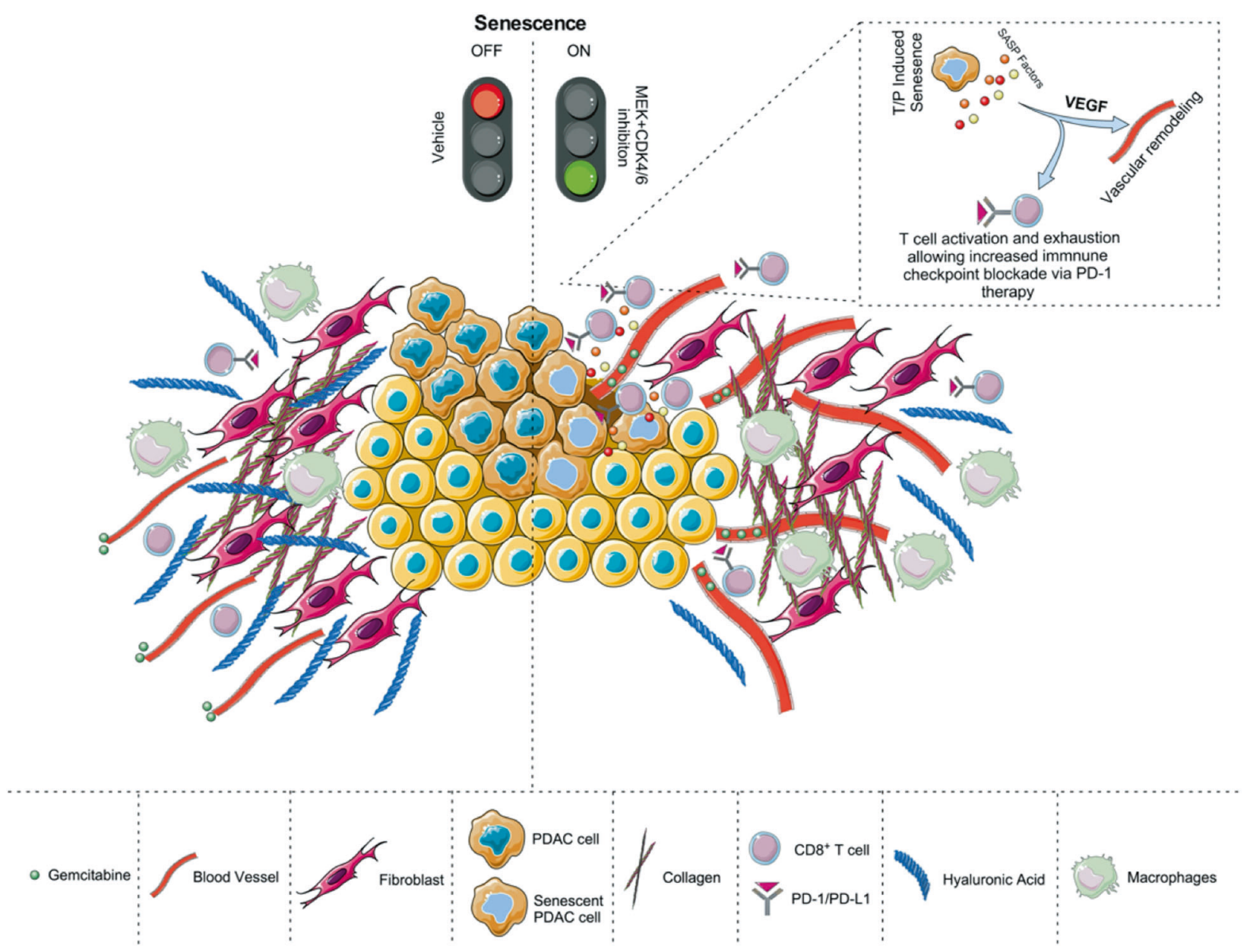

Fig. 1 Senescence creates therapeutic liabilities for chemo- and immunotherapy in PDAC. Trametinib (T) and palbociclib (P) combo treatment induces SASP factors, which enhance vascular remodeling and decrease hyaluronic acid texture without affecting collagen deposition in TME. Vascular remodeling in PDAC allows increased vascular perfusion, causing enhanced gemcitabine delivery. At the same time, increased vessel density and endothelial cell activation permits escalated $\mathrm{CD}^{+} \mathrm{T}$ cell infiltration. Combo treatment-induced SASP factors boost $T$ cell activation and exhaustion, enabling PD-1/PD-L1 axis susceptible to immunotherapy.

\section{REFERENCES}

1. Lee, S. \& Schmitt, C. A. Nat. Cell Biol. 21, 94-101 (2019).

2. Wang, C. et al. Nature 574, 268-272 (2019).

3. Lee, K. E. \& Bar-Sagi, D. Cancer Cell. 18, 448-458 (2010).

4. Morton, J. P. et al. Proc. Natl. Acad. Sci. USA 107, 246-251 (2010).

5. Guerra, C. et al. Cancer Cell. 19, 728-739 (2011).
6. Caldwell, M. E. et al. Oncogene 31, 1599-1608 (2012).

7. Lesina, M. et al. J. Clin. Invest. 126, 2919-2932 (2016).

8. Ruscetti, M. et al. Cell 181, 424-441 (2020).

9. Ozdemir, B. C. et al. Cancer Cell. 25, 719-734 (2014).

10. Hingorani, S. R. et al. J. Clin. Oncol. 36, 359-366 (2018).

11. Weinberg, R. A. One Renegade Cell: How Cancer Begins. (Basic Books, 1998). 